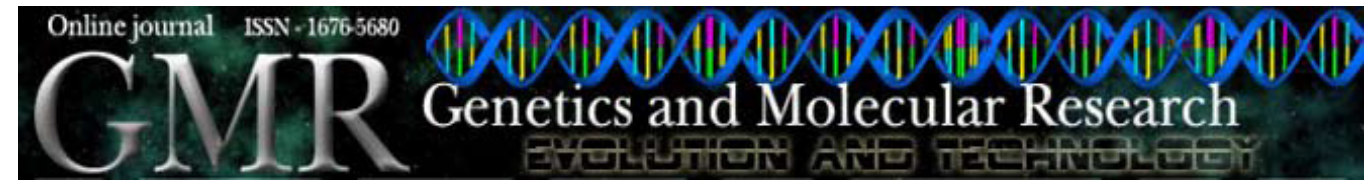

\title{
Global gene expression profile in myelodysplastic syndromes using SAGE
}

\author{
C.F. Mendiburu ${ }^{1}$, W.A. Silva Jr. ${ }^{2}$, O. Ricci Jr. ${ }^{3}$, C.R. Bonini-Domingos ${ }^{1}$ \\ and A.C. Fett-Conte ${ }^{4}$ \\ ${ }^{1}$ Departamento de Biologia, Instituto de Biociência Letras e Ciências Exatas, \\ Universidade Estadual de São Paulo, São José do Rio Preto, SP, Brasil \\ ${ }^{2}$ Departamento de Genética, Faculdade de Medicina de Ribeirão Preto, \\ Universidade de São Paulo, Ribeirão Preto, SP, Brasil \\ ${ }^{3}$ Departamento de Medicina, Faculdade de Medicina de São José do Rio Preto, \\ São José do Rio Preto, SP, Brasil \\ ${ }^{4}$ Departamento de Biologia Molecular, \\ Faculdade de Medicina de São José do Rio Preto, \\ São José do Rio Preto, SP, Brasil \\ Corresponding author: C.F. Mendiburu \\ E-mail: fmendiburu@yahoo.com.ar
}

Genet. Mol. Res. 7 (4): 1245-1250 (2008)

Received September 3, 2008

Accepted October 1, 2008

Published November 11, 2008

\begin{abstract}
The molecular pathogenesis of myelodysplastic syndromes (MDS) is poorly understood. In order to expand our knowledge of genetic defects in MDS, we determined the overall profile of genes expressed in bone marrow from patients with refractory anemia with excess blasts (RAEB) by serial analysis of gene expression (SAGE). The present report describes a partial transcriptome of RAEB bone marrow derived from 56,694 sequenced tags that provides information about expressed gene products. This is the first attempt to determine an overall profile of gene expression specifically in RAEB at diagnosis
\end{abstract}


using SAGE, which should be useful in the understanding of the physiopathology of MDS and in identifying the genes involved.

Key words: Serial analysis of gene expression methodology; Myelodysplastic syndrome; Gene expression profile; Refractory anemia with excess blasts

\section{INTRODUCTION}

Myelodysplastic syndromes (MDS) are a group of hematological disorders characterized by peripheral cytopenias with a high risk of progression to acute myeloid leukemia (AML) (Hellström-Lindberg and Malcovati, 2008). MDS mainly affects the elderly. Recently, an annual incidence of 5.4-36.2/100,000 was reported in the United States in individuals between 60 and 84 years old (Ma et al., 2007).

The clinical course of MDS can be divided into several stages. After a variable interval, about $10-40 \%$ of the patients in the early indolent chronic stage may progress to an advanced stage of refractory anemia with excess blasts (RAEB) and subsequently to AML (Mano, 2006).

Although MDS has been recognized as an important disease for more than 50 years, its molecular pathogenesis and the basis for its leukemic progression remain undefined (Look, 2005). Several genes, including oncogenes, cell cycle regulatory genes, apoptotic genes and genes that regulate DNA methylation and histone deacetylation, have been implicated in the molecular pathogenesis of MDS. The application of cDNA microarray technology has facilitated the detection of previously unidentified genes, demonstrating the potential of genome-wide approaches in investigating the molecular pathogeneses of MDS (Nishino and Chang, 2005).

As serial analysis of gene expression (SAGE) is a powerful tool to obtain comprehensive and quantitative gene expression profiles from cell populations (Velculescu et al., 1995), we performed the first analyses using SAGE on bone marrow cells from patients with the RAEB subtype of MDS, in order to expand our knowledge of genetic defects in this disease. The present report describes a partial transcriptome of bone marrow from patients with RAEB.

\section{MATERIAL AND METHODS}

\section{Bone marrow samples}

The study was approved by the Research Ethics Committee of the Institute of Biosciences, Letters and Exact Sciences, State University of São Paulo - UNESP, and written informed consent was obtained from all patients.

Bone marrow samples were obtained from three male and three female adult patients (aged 26 to 68 years old; mean [SD] age, 52 [17.25] years) with primary RAEB with diagnosis based on the FAB criteria (Bennett et al., 1982). Only samples from patients with a normal karyotype in cytogenetic analysis were included. Patients were seen in the Hematology and Hemotherapy Service of the University Hospital of São José do Rio Preto, State of São Paulo, Brazil. After aspiration, fresh bone marrow samples were treated with red blood cell lysis solution, and the nucleated cell pellet was resuspended in PBS and Trizol (Invitrogen, USA) and stored at $-80^{\circ} \mathrm{C}$ until nucleic acid extraction. 


\section{RNA extraction and pooling}

Total RNA was extracted using Trizol following the protocol supplied by the manufacturer. To ensure that the gene expression measured by the SAGE assay was not affected by degradation of the RNA extracted, gel electrophoresis and spectrophotometer readings were used to evaluate the quality and quantity of the RNA, respectively. Equal amounts of RNA from each patient were mixed to generate a pool of total RNA.

\section{Serial analysis of gene expression}

An RAEB bone marrow SAGE library was constructed using $27 \mu \mathrm{g}$ of the total RNA pool using the I-SAGETM Kit (Invitrogen, USA) and strictly following the manufacturer protocol. The key steps included the following: poly $(\mathrm{A}+)$ mRNAs in the sample were captured by oligo $(\mathrm{dT})$ magnetic beads to synthesize cDNA; the cDNA was digested with NlaIII (anchoring enzyme) and 3' cDNA isolated by the magnetic beads; the resultant 3' cDNA was split into two fractions and ligated to two SAGE adapters, A and B; SAGE tags were released by the tagging enzyme BsmFI blunt ended with the Klenow polymerase fragment, and the tags from the two fractions were ligated to form $\sim 100$-bp ditags; a 1:120 dilution of the ligation product was amplified in 200 polymerase chain reactions (PCR); precipitated PCR products were run on a $12 \%$ polyacrylamide gel electrophoresis (PAGE), and only the 100-bp band containing ditags was isolated and digested with NlaIII; the products of the digestion were run on a 12\% PAGE and the 26-bp bands containing ditags purified and used for self-ligation to form concatamers; these were run on an $8 \%$ PAGE and a fraction from 500 to 1000 bp was isolated and cloned into a pZEro-1 vector digested with $S p h I$; cloned concatamers were used as templates for sequencing reactions. Sequencing reactions were carried out using DYEnamic ET Dye Terminator Sequencing Kit (Amersham Biosciences, USA) and a MegaBace 1000 DNA sequencer (Amersham Biosciences, USA).

\section{Data analysis}

Sequence files were analyzed with the SAGE2000 software, which removes the two tags flanked by NlaIII sites. Gene identification and UniGene cluster assignment of each tag was obtained using the "reliable" tag-to-gene map from the SAGEmap NCBI site (http://www. ncbi.nlm.nih.gov/). The extracted tags were uploaded to the SAGEmap and corresponding accession numbers were retrieved using the Homos sapiens NCBI-GenBank database. The FatiGO+ web tool was used for functional analysis of expressed genes based on the Gene Ontology database (Al-Shahrour et al., 2007).

\section{RESULTS}

The RAEB bone marrow SAGE library denoted SAGE_BoneMarrow_MDS_RAEB was deposited in the Genome Data Mining Website (http://gdm.fmrp.usp.br/h2g/library/1153) of the Molecular Genetics and Bioinformatics Laboratory. This SAGE library yielded 56,694 sequenced tags, of which 16,244 corresponded to unique tags. Among the unique tags, 10,728 were from annotated genes and 5516 were no-matches with gene sequences. Table 1 summa- 


\begin{tabular}{lcc}
\multicolumn{4}{l}{ Table 1. General serial analysis of gene expression (SAGE) data statistics of library obtained. } \\
\hline Origin & Frequency & Unique tags \\
\hline All & 56,694 & 16,244 \\
Annotated & 47,776 & 10,728 \\
EST/cDNA & 315 & 212 \\
Ribosomal & 8,693 & 269 \\
No-matches & 8,918 & 5,516 \\
\hline Frequency distribution $^{\text {a }}$ & No. of tags sequenced (\%) $^{\text {o }}$ & No. of unique tags (\%) \\
\hline$>200$ tags & $12,761(22.5 \%)$ & $29(0.2 \%)$ \\
$100-200$ tags & $5,722(10.1 \%)$ & $40(0.3 \%)$ \\
$20-99$ tags & $8,530(15.0 \%)$ & $216(1.3 \%)$ \\
$5-19$ tags & $9,810(17.3 \%)$ & $1,177(7.2 \%)$ \\
$2-4$ tags & $8,463(15.0 \%)$ & $3,374(20.8 \%)$ \\
1 tag & $11,408(20.1 \%)$ & $11,408(70.2 \%)$ \\
Total & $56,694(100 \%)$ & $16,244(100 \%)$ \\
\hline
\end{tabular}

${ }^{a}$ Calculation of the frequency distribution of a given tag was based on total unique tags sequenced in the library. ${ }^{\mathrm{b}} \mathrm{Some}$ genes have more than one tag.

rizes the general statistical data of this library.

Among the unique tags, 12,761 (22.5\%) tags corresponding to 29 genes showed frequencies $>200$ and 32,525 (57.4\%) tags of the 4807 genes had frequencies between 2 and 200. Tags with a frequency of one represented $70.2 \%$ of the unique tags; however, these were not analyzed because they likely represent artifacts of sequencing or of the SAGE procedure.

The 20 most expressed genes in the RAEB bone marrow SAGE library are shown in Table 2. Eight (40\%) of these 20 most expressed genes correspond to ribosomal genes, while the

Table 2. The 20 most expressed genes in the refractory anemia with excess blast (RAEB) bone marrow serial analysis of gene expression (SAGE) library.

\begin{tabular}{rrrrrll}
\hline & Tag sequence & Tags & TPM & UniGene ID & Gene & Description \\
\hline 1 & TACCTGCAGA & 2050 & 36080 & Hs.416073 & S100A8 & S100 calcium-binding protein A8 \\
2 & GTGGCCACGG & 1336 & 23514 & Hs.112405 & S100A9 & S100 calcium-binding protein A9 \\
3 & GCCTGCTATT & 1089 & 19166 & Hs.380781 & DEFA1 & Defensin, alpha 1 \\
4 & CTTCTTGCCC & 656 & 11546 & Hs.654744 & HBA2 & Hemoglobin, alpha 2 \\
5 & GAGGGAGTTT & 480 & 8448 & Hs.523463 & RPL27A & Ribosomal protein L27a \\
6 & GCAAGAAAGT & 478 & 8413 & Hs.523443 & HBB & Hemoglobin, beta \\
7 & GTTGTGGTTA & 443 & 7797 & Hs.534255 & B2M & Beta-2-microglobulin \\
8 & GGATTTGGCC & 406 & 7146 & Hs.437594 & RPLP2 & Ribosomal protein, large, P2 \\
9 & GGGCTGGGGT & 363 & 6389 & Hs.425125 & RPL29 & Ribosomal protein L29 \\
10 & ATGTAAAAAA & 344 & 6054 & Hs.706744 & LYZ & Lysozyme (renal amyloidosis) \\
11 & TAGGTTGTCT & 328 & 5773 & Hs.374596 & TPT1 & Tumor protein, translationally controlled 1 \\
12 & CTGGGTTAAT & 308 & 5421 & Hs.438429 & RPS19 & Ribosomal protein S19 \\
13 & CCCAACGCGC & 302 & 5315 & Hs.654744 & HBA2 & Hemoglobin, alpha 2 \\
14 & GAAATAAAGC & 300 & 5280 & Hs.510635 & IGHG1 & Immunoglobulin heavy \\
& & & & & constant gamma 1 (G1m marker) \\
15 & TGCACGTTTT & 293 & 5157 & Hs.265174 & RPL32 & Ribosomal protein L32 \\
16 & CCCATCGTCC & 289 & 5086 & Hs.559716 & - & Transcribed locus, moderately similar to NP_536846.1 \\
& & & & & cytochrome c oxidase subunit II [Homo sapiens] \\
17 & ATAATTCTTT & 286 & 5034 & Hs.156367 & RPS29 & Ribosomal protein S29 \\
18 & TTGGTCCTCT & 282 & 4963 & Hs.632703 & RPL41 & Ribosomal protein L41 \\
19 & AGGGCTTCCA & 279 & 4910 & Hs.534404 & RPL10 & Ribosomal protein L10 \\
20 & GAGCCCAGCC & 276 & 4858 & Hs.654826 & SPATS2 & Spermatogenesis associated, serine-rich 2 \\
\hline aTPM $=$ tags per million; [ Tag frequency)(1,000,000)/Total No. of sequenced tags].
\end{tabular}


most expressed tags correspond to two calcium-binding protein genes (S100A8 and S100A9).

To obtain better knowledge of the functional categories of the overall gene expression profile, 1673 genes with tag frequencies $\geq 3$ were analyzed in the FatiGO+ web tool. Figure 1 illustrates the distribution of expressed genes in 21 principal functional categories (with number of genes $>1 \%$ ) as defined by the Gene Ontology (GO) Consortium. The most common transcripts correspond to genes involved in cellular metabolism. On the other hand, genes involved in other processes such as cytokine production, cell division, cellular homeostasis, and cell activation were not so frequently expressed.

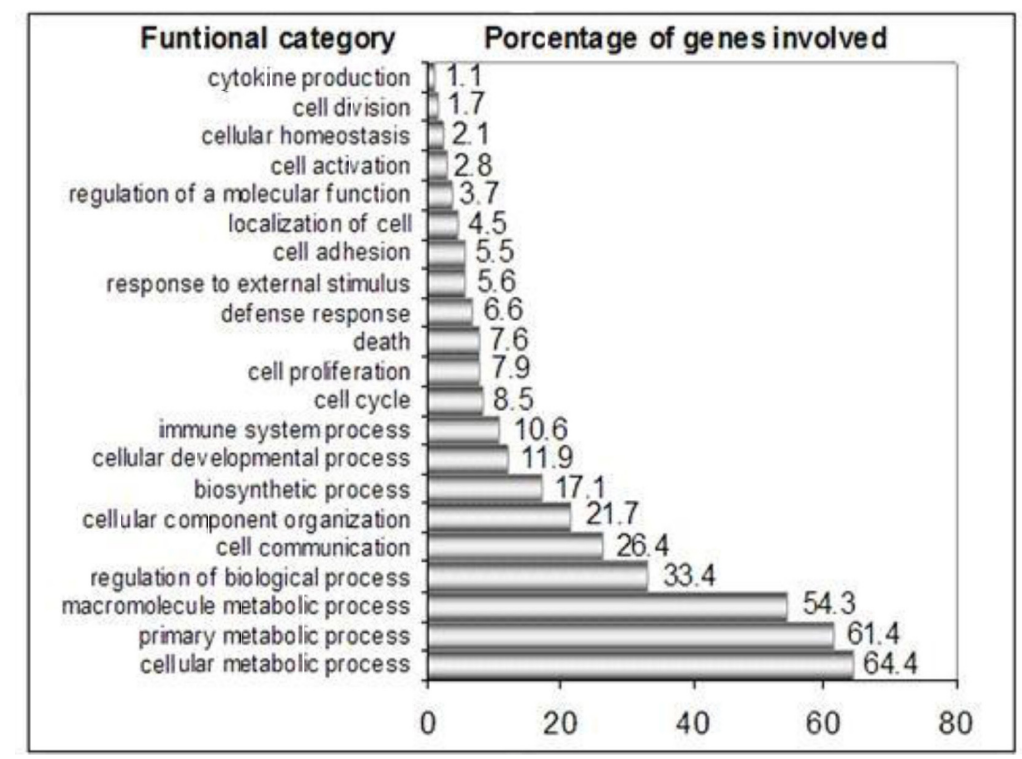

Figure 1. Functional categories assigned to individual genes identified in the refractory anemia with excess blast (RAEB) bone marrow serial analysis of gene expression (SAGE) library.

\section{DISCUSSION}

The molecular pathogenesis of MDS remains uncertain. The lack of suitable experimental models for MDS hampers progress in understanding the biology of this disease. New approaches to elucidate the underlying abnormalities are required (Hofmann et al., 2002). Hence, we proposed the first attempt to determine an overall profile of gene expression specifically in RAEB at diagnosis using the SAGE methodology.

Since its establishment, SAGE technology has been successfully and widely applied in transcript profile studies in biology and oncology to characterize the genes that are responsible for observed biological phenotypic changes (Velculescu et al., 1995; Harbers and Carninci, 2005). Unlike microarray technology, SAGE can be performed on very few cells, and does not require a priori knowledge of the transcriptome of a given tissue (Velculescu et al., 1995). This opens the opportunity to discover novel genes involved in the pathogenesis of MDS.

All transcripts expressed with an NIaIII site can be "tagged" and counted efficiently in 
large numbers (typically $>50,000$ tags) using automated sequencing (Boon et al., 2002). With this in mind, we considered the 52,309 tags from RAEB bone marrow to be a reasonable number.

GO provides a tool for functional interpretation of expressed genes in RAEB. We classified the functional category of 1673 RAEB expressed genes by GO. These genes were classified into 21 major functional categories. The comparison of this functional profile with that of normal bone marrow may elucidate the biological processes affected in RAEB.

\section{CONCLUSION}

Because SAGE provides a qualitative and quantitative evaluation of messenger RNA abundance, our data provide an estimation of the genes expressed in RAEB bone marrow cells. Data generated by the SAGE library will help to identify genes and molecular processes involved in the etiology and/or progression of RAEB. This knowledge will be useful in understanding the physiopathology of MDS as well as in identifying possible diagnostic and prognostic biomarkers and potential therapeutic targets.

\section{ACKNOWLEDGMENTS}

We thank all patients who collaborated with this study. We also thank Dr. Carlos Maluf for the collection of bone marrow samples, Daniel G. Pinheiro for his help with bioinformatics analysis, Anemarie R. Dinarte for technical support, and David A. Hewitt for English correction and idiomatic adaptations. C.F. Mendiburu is grateful to the Brazilian agency Coordenação de Aperfeiçoamento de Pessoal de Nível Superior (CAPES) for a Ph.D. scholarship.

\section{REFERENCES}

Al-Shahrour F, Minguez P, Tarraga J, Medina I, et al. (2007). FatiGO +: a functional profiling tool for genomic data. Integration of functional annotation, regulatory motifs and interaction data with microarray experiments. Nucleic Acids Res. 35: W91-W96.

Bennett JM, Catovsky D, Daniel MT, Flandrin G, et al. (1982). Proposals for the classification of the myelodysplastic syndromes. Br. J. Haematol. 51: 189-199.

Boon K, Osorio EC, Greenhut SF, Schaefer CF, et al. (2002). An anatomy of normal and malignant gene expression. Proc. Natl. Acad. Sci. U.S.A. 99: 11287-11292.

Harbers M and Carninci P (2005). Tag-based approaches for transcriptome research and genome annotation. Nat. Methods 2: 495-502.

Hellström-Lindberg E and Malcovati L (2008). Supportive care, growth factors, and new therapies in myelodysplastic syndromes. Blood Rev. 22: 75-91.

Hofmann WK, de Vos S , Komor M, Hoelzer D, et al. (2002). Characterization of gene expression of CD34+ cells from normal and myelodysplastic bone marrow. Blood 100: 3553-3560.

Look AT (2005). Molecular Pathogenesis of MDS. Hematology Am. Soc. Hematol. Educ. Program, 156-160.

Ma X, Does M, Raza A and Mayne ST (2007). Myelodysplastic syndromes: incidence and survival in the United States. Cancer 109: 1536-1542.

Mano H (2006). DNA micro-array analysis of myelodysplastic syndrome. Leuk. Lymphoma 47: 9-14.

Nishino HT and Chang CC (2005). Myelodysplastic syndromes: clinicopathologic features, pathobiology, and molecular pathogenesis. Arch. Pathol. Lab. Med. 129: 1299-1310.

Velculescu VE, Zhang L, Vogelstein B and Kinzler KW (1995). Serial analysis of gene expression. Science 270: 484-487. 